
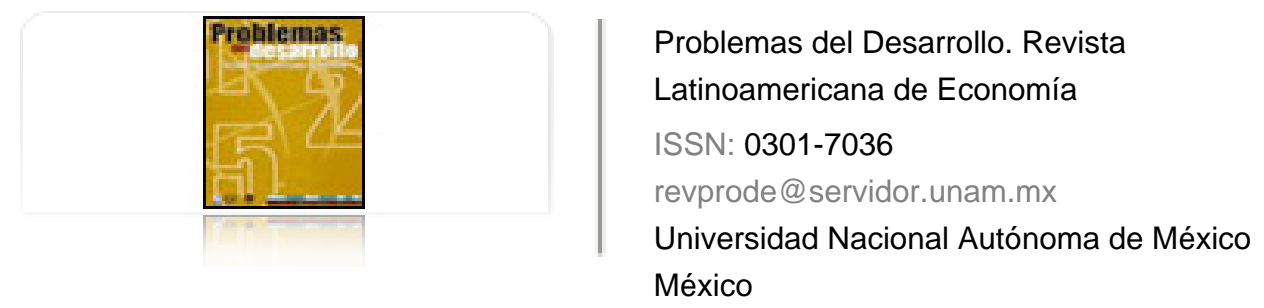

Amarante, Verónica; Espino, Alma

INFORMALIDAD Y DESPROTECCIÓN SOCIAL EN URUGUAY

Problemas del Desarrollo. Revista Latinoamericana de Economía, vol. 40, núm. 158, julio-septiembre, 2009, pp. 33-54

Universidad Nacional Autónoma de México

Distrito Federal, México

Disponible en: http://www.redalyc.org/articulo.oa?id=11820200002

- Cómo citar el artículo

- Número completo

- Más información del artículo

Página de la revista en redalyc.org

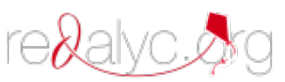

Sistema de Información Científica

Red de Revistas Científicas de América Latina, el Caribe, España y Portugal Proyecto académico sin fines de lucro, desarrollado bajo la iniciativa de acceso abierto 


\title{
INFORMALIDAD Y DESPROTECCION SOCIAL EN URUGUAY
}

\author{
Verónica Amarante* \\ Alma Espino**
}

Fecha de recepción: 19 de febrero de 2009. Fecha de aceptación: 5 de julio de 2009.

\section{Resumen}

Este trabajo cuantifica la incidencia de la informalidad-definida de acuerdo con el criterio de la Organización Internacional del Trabajo- y la desprotección social en Uruguay en el periodo 1991-2005, analiza su superposición y las características y remuneraciones de los trabajadores involucrados. La cotización a la seguridad social resulta determinante en el ordenamiento de los trabajadores según sus ingresos. Se fortalece así la idea de que la categoría de desprotección social resulta más adecuada para el estudio de la calidad del trabajo en Uruguay. Sin embargo, se evidencia la necesidad de contar con mejores herramientas metodológicas para realizar un estudio más amplio sobre la temática de calidad del empleo, que abarque otras dimensiones. Palabras clave: empleo, informalidad, protección social, mercado laboral, trabajo.

\begin{abstract}
This study quantifies the incidence of informality -defined according to the criterion used by the International Labor Organization -and the lack of social security in Uruguay during the period 1991-2005. It analyzes its superimposition and the characteristics and remunerations of the workers involved. Social security payments are the determinant factor in categorizing the workers according to their income levels. This strengthens the idea that the category lacking social security is more suitable for studying labor quality in Uruguay. However, the need for better methodological tools is clear, if we are to realize a broader study of the question of job quality, embracing other dimensions.
\end{abstract}

Keywords: employment, informality, social security, labor market, work.

* Investigadora en el Instituto de Economía, Universidad de la República, Uruguay. Correo electrónico: vero@iecon.ccee.edu.uy

** Licenciada en Economía, Facultad de Economía, UNAM. Instituto de Economía, Facultad de Ciencias económicas y de Administración. Universidad de la República, Uruguay.

Correo electrónico: alma@iecon.ccee.edu.uy 


\section{Résumé}

Ce travail quantifie l'incidence de l'économie informelle - définie selon le critère de l'Organisation Internationale du Travail - et de la déprotection sociale en Uruguay durant la période 1991-2005, analyse leur conjonction et les caractéristiques et rémunérations des travailleurs concernés. La cotisation à la sécurité sociale s'avére déterminante pour le classement des travailleurs selon leurs revenus. On voit ainsi renforcée l'idée selon laquelle la catégorie de déprotection sociale s'avére plus adéquate pour l'étude de la qualité du travail en Uruguay. Néanmoins, il est mis en évidence le besoin de pouvoir compter sur de meilleurs outils méthodologiques pour réaliser une étude plus vaste sur la thématique de la qualité de l'emploi, qui engloberait d'autres dimensions.

Mots clés: emploi, économie informelle, protection sociale, marché du travail, travail.

\section{Resumo}

Este trabalho quantifica a incidência da informalidade -definida de acordo com o critério da Organização Internacional do Trabalho-e a desproteção social no Uruguai no período 1991-2005, analisa a sua superposição e as características e remunerações dos trabalhadores envolvidos. A cotização para a seguridade social resulta determinante na ordenação dos trabalhadores segundo seus ingressos. Fortalece-se assim a idéia de que a categoria de desproteção social resulta mais adequada para o estudo da qualidade do trabalho no Uruguai. No entanto, evidencia-se a necessidade de contar com melhores ferramentas metodológicas para realizar um estudo mais amplo sobre a temática de qualidade do emprego, que abarque outras dimensões.

Palavras-chave: emprego, informalidade, proteção social, mercado de trabalho, trabalho. 


\section{Introducción}

\section{$\mathcal{L}$} a coexistencia de puestos de trabajo de alta y baja calidad ha sido un tema central de análisis de las ciencias sociales, especialmente en los países de América Latina. En este artículo se revisan los principales enfoques que explican el empleo informal, con el objetivo de entender la problemática del mercado laboral en Uruguay y sus especificidades. Se cuantifica la incidencia de la informalidad -definida de acuerdo con el criterio de la Organización Internacional del Trabajo- y la desprotección social en el periodo 1991-2005, se analiza su superposición y las características y remuneraciones de los trabajadores involucrados.

\section{Los distintos enfoques sobre la informalidad}

Uno de los aspectos centrales que ha intentado explicar la economía, específicamente en las áreas de desarrollo y economía laboral, es la coexistencia de puestos de trabajo de alta y baja calidad en una misma economía y en un mismo momento. En América Latina este problema ha sido abordado desde tres enfoques principales que a continuación se presentan sintéticamente ${ }^{1}$.

Desde las teorías surgidas en países desarrollados, uno de los alejamientos de la tradición neoclásica que ha tenido más influencia, se encuentra en las teorías de mercados laborales segmentados o duales. Éstas se engloban dentro del enfoque institucionalista. En términos sintéticos, se asume que el comportamiento maximizador de los empresarios los conduce a buscar estrategias reductoras de costos, que se traducen en nuevas formas contractuales que dan origen a empleos de baja calidad, que suelen asociarse al concepto de empleos informales. El origen de este enfoque se ubica en los trabajos pioneros de Piore (1975), que recibieron acerbas criticas fuertemente por sus debilidades teóricas y empíricas (Cain, 1976). Más recientemente, estas teorías resurgen al ser retomadas por economistas que analizan los mercados laborales duales utilizando recursos metodológicos más modernos e incorporando la teoría de información imperfecta (Bulow y Summers, 1986; Saint Paul, 1996; entre otros). Diversos trabajos empíricos se insertan en este marco y desarrollan pruebas econométricas para analizar la existencia de esta segmentación o dualidad (Dickens y Lang 1985; Maloney, 1998; entre otros).

1 Se sigue el ordenamiento propuesto en Cimoli et al., 2005; Lay, 2003; Filgueira y Gelber, 2003; Bucheli, 2002, entre otros, aunque debe advertirse que no hay un consenso sobre el agrupamiento de los distintos autores y enfoques. 
Los trabajos de Portes (Portes, Castells y Benton, 1989; Portes, 1995; entre otros), han sido identificados por algunos autores dentro de una perspectiva neomarxista, puesto que señalan que las dinámicas perversas de mercado surgen como elemento integral de la estrategia de acumulación de las empresas modernas. Para estos autores, las actividades informales son procíclicas, en cuanto están íntegramente ligadas al funcionamiento del sector moderno como parte de una misma economía.

Portes y Haller (2004) definen tres tipos de actividades informales -cuyo punto de coincidencia es estar fuera del ámbito de la regulación del Estado-: de supervivencia, explotación dependiente y crecimiento. Las primeras tienen como finalidad la supervivencia de una persona o de un hogar mediante la producción directa con fines de subsistencia o de la mera venta de bienes y servicios en el mercado; las segundas están orientadas a mejorar la flexibilidad de la gestión y reducir los costos laborales de las empresas del sector formal por medio de la contratación "fuera de plantilla" y la subcontratación de empresarios informales; las últimas pueden estar organizadas por pequeñas empresas con el objetivo de acumular capital, aprovechando las relaciones de solidaridad que puedan existir entre ellas, la mayor flexibilidad y los menores costos.

El segundo enfoque corresponde a la corriente estructuralista del pensamiento económico latinoamericano. El concepto de heterogeneidad estructural desarrollado por Pinto $(1965,1970)$ implica la coexistencia de un sector de alta productividad orientado a la exportación y un conjunto de actividades de baja productividad, que opera fundamentalmente para el mercado doméstico. Dentro de ese marco, la Organización Internacional del Trabajo (OIT) ha impulsado el concepto sector informal, compuesto por actividades marginales -distintas y no relacionadas con el sector formal- que proveen ingresos y redes de seguridad para los pobres durante las crisis (OIT, 1972; Tokman, 1978).

En esta interpretación se enfatiza la importancia de considerar distintos segmentos del mercado laboral, cuyas diferencias se asocian a las productividades del trabajo y a la incorporación de la tecnología. La informalidad refiere así a trabajos de baja productividad en segmentos marginales de la economía y a unidades económicas de subsistencia, con escasa o nula capacidad de acumulación. En consecuencia, el concepto sector informal se asocia a los ocupados con ingresos insuficientes en actividades de baja productividad pero funcionales al resto de la economía. La persistencia de las actividades informales se debe a la falta de demanda o de oportunidades laborales en el sector formal o moderno, lo cual crea un excedente de mano de obra, ya sea por el escaso crecimiento económico o por el rápido crecimiento demográfico. Las características típicas del trabajo informal que suelen destacarse, se refieren a negocios de tamaño pequeño, que utilizan tecnologías simples, con poca dotación de capital físico, 
con escasa división en la propiedad de los medios de producción, y que generalmente operan fuera del marco legal institucional (Tokman, 2001).

Finalmente, el enfoque legalista - del cual De Soto (1987) es el principal representante en América Latina- sostiene que el sector informal se compone de microempresarios que eligen operar de esa manera para reducir los costos, tiempo y esfuerzos que supone el registro formal. Las causas de la informalidad se atribuyen a un marco legal que impone reglas que exceden el marco normativo socialmente aceptable. Y la informalidad se define como el conjunto de actividades ilegales no antisociales (antisociales serían el narcotráfico o el secuestro); se trata de un sector cuyas fronteras con el mundo legal están poco definidas. Su origen, por tanto, deriva de la excesiva regulación en el mercado de trabajo, que impone restricciones tanto en la contratación como en el ámbito de las remuneraciones, desestimulando el crecimiento de la actividad y de la demanda de trabajo, y dando lugar a la proliferación de empresas con producción intensiva en capital, así como a la propensión a evadir los controles y normas del mercado laboral.

A pesar de las diferencias respecto a la interpretación de la causalidad de los fenómenos, los tres enfoques parten de la existencia de distintos segmentos del mercado laboral o, lo que es lo mismo, de la coexistencia de trabajos de alta y baja calidad. El enfoque adoptado y, por lo tanto, la explicación subyacente sobre la problemática del mercado laboral, resulta fundamental en el momento de discutir políticas públicas adecuadas. También será relevante para la cuantificación, ya que como se desarrolla en el siguiente apartado, cada enfoque conduce a una definición operativa distinta.

\section{La medición de la calidad del empleo}

Se han propuesto distintas definiciones operativas de trabajo informal, enmarcadas en los enfoques antes descritos. Portes (1995) señala que la medida ideal del peso del sector informal sería el número de horas utilizadas en el trabajo regulado en relación con las del no regulado. También acepta la proporción de la fuerza laboral excluida de la cobertura legal relativa al empleo, como protección contra despidos arbitrarios, compensación por desempleo, seguro por accidentes, ausencias pagadas y pensiones por jubilación. De esta manera, la falta de protección social podría ser un indicador utilizado en el marco del enfoque de Portes.

En la línea del enfoque estructural, la OIT, a través del Programa Regional del Empleo para América Latina y el Caribe (PREALC), ha realizado diversos esfuerzos para la cuantificación del fenómeno. Durante la década de 1990, los organismos en- 
cargados de la revisión IV de los Sistemas de Contabilidad Nacional (OIT, OCDE, FMI, UNSDY BM) decidieron adoptar un concepto coordinado de sector informal. Así, en la XV Conferencia Internacional de Estadísticos del Trabajo (CIET), convocada por la OIT en 1993, se delimitó el concepto de trabajo en el sector informal, que luego fue incorporado al Sistema de Cuentas Nacionales Rev. 1993. Esta delimitación del concepto se tradujo, en la mayoría de los países e incluso en las propias mediciones realizadas por la OIT, en la cuantificación del empleo informal como el que abarca una o más de las siguientes categorías: servicio doméstico, autoempleo -excluyendo administradores y profesionales-, trabajo familiar no remunerado y trabajo en pequeñas empresas que tienen menos de cinco empleados. Sin embargo, la delimitación operativa no fue estricta: se brindaron lineamientos generales y se concedió flexibilidad a los países para definir y medir el sector informal. Los principales aspectos en los que las prácticas nacionales para la medición del trabajo informal pueden diferir, refieren a las ramas de actividad abarcadas (específicamente respecto de la inclusión de la agricultura), el criterio para definir el sector informal (se puede considerar el tamaño de las empresas o el registro de la empresa o el trabajador), la inclusión de los trabajadores domésticos remunerados y la inclusión de las personas que tienen un trabajo secundario en el sector informal, pero cuyo empleo principal no pertenece a ese sector. Es decir, estos criterios permiten considerar a los trabajadores no protegidos por la seguridad social, pero no necesariamente los incluyen. Y la principal adaptación, que es la medición realizada por la OIT, no relaciona de manera directa la informalidad con la desprotección social.

Posteriormente, y en reconocimiento de la necesidad de complementar la definición y medición del empleo en el sector informal, la XVII CIET propone una nueva definición. Se distingue entre el empleo en el sector informal y otros empleos informales fuera del sector, definiendo a dicho sector de acuerdo con las características de las unidades productivas y no de los trabajadores. Además, a la medición tradicional se agrega el empleo informal en unidades productivas del sector formal. Así, se diferencian tres grupos de unidades de producción: empresas del sector formal, empresas del sector informal y hogares. Los hogares como unidades de producción son aquellos que producen bienes para su consumo (por ejemplo, construcción de su vivienda) o que emplean trabajadores domésticos remunerados (empleados del hogar, lavanderas, jardineros, cuidadores, choferes, etcétera) ${ }^{2}$. El empleo informal comprende a los trabajadores por cuenta propia y a los empleadores dueños de sus

2 Hasta la XV CIET, los hogares que empleaban a trabajadores domésticos remunerados se incluían en el sector informal. 
empresas en el sector informal; a los trabajadores familiares auxiliares sin contrato; a los asalariados que tienen puestos de trabajo informal, ya sea en empresas del sector formal, informal u hogares, "si su relación de trabajo no se rige por la legislación laboral común”, no tributan ni tienen protección, ya sea porque no estén registrados los trabajadores o las empresas; puestos localizados fuera de la empresa o trabajos de corta duración, etc.; miembros de cooperativas informales; trabajadores en la producción de bienes para su propio hogar.

Esto implica un cambio en la definición tradicional y un mayor acercamiento al concepto de protección social, aunque esta nueva definición no se ha instrumentado aún de manera extendida. El Panorama Laboral para América Latina y el Caribe de la OIT disponible (2005) no incorpora todavía esta nueva manera de cuantificar la informalidad, y sigue con el criterio tradicional basado en la XV CIET ${ }^{3}$.

Finalmente, en relación con la perspectiva de De Soto, cabe señalar que aunque su enfoque es usado en distintos estudios sobre informalidad, es más una referencia teórica que empírica, por lo que en este caso no se puede identificar una medida operativa concreta (Bucheli, 2002).

En este documento se analiza la magnitud y evolución del fenómeno de la informalidad en su acepción tradicional (PREALC, OIT) y también de la desprotección social, entendida como la falta de aportaciones a la seguridad social. El análisis se basa en la información provista por la Encuesta Continua de Hogares $(\mathrm{ECH})$ relevada por el Instituto Nacional de Estadística (INE) para el periodo 1991-2005 . Se cuantifican ambos fenómenos y se analiza su vinculación, con el objetivo de identificar el conjunto de trabajadores que podrían presentar las situaciones más desventajosas tanto en términos de ingresos como de cobertura de la seguridad social. Para tales efectos, a este segmento de la fuerza de trabajo se le denomina vulnerables.

El empleo informal considera a los trabajadores del servicio doméstico, los familiares no remunerados, los trabajadores privados en establecimientos con menos de cinco empleados y los trabajadores por cuenta propia con y sin local, excluyendo administrativos, profesionales y técnicos (OIT, XV CIET). Para la cuantificación de la desprotección social, se consideran dos aproximaciones: una cuantificación indirecta, a través de la información sobre cobertura de salud que surge de la ECH y, a partir de 2001, sobre derechos jubilatorios.

3 En 1997, la OIT lanzó el Programa de Empleo Decente, concepto identificado con cuatro elementos: empleo, protección social, derechos de los trabajadores y diálogo social.

4 Esta encuesta es representativa de las localidades urbanas de 5000 y más habitantes, donde se concentra el $85 \%$ de la población del país. 


\section{Informalidad y desprotección social en Uruguay}

Los primeros trabajos que analizan y cuantifican el sector informal en Uruguay datan de finales de la década de 1980. La mayor parte se basa en la definición tradicional de PREALC, incluso aquellos que comparten las contribuciones conceptuales de Portes (Diez de Medina y Gerstenfeld, 1986; Groskoff y Melgar, 1990; Portes, Blitzer y Curtis, 1986) ${ }^{5}$. Entre los trabajos más recientes destacan Notaro, 2005; Amarante y Arim, 2005a, 2005b; y Bucheli, 2002.

\section{Evolución de la informalidad}

Desde una perspectiva de largo plazo, la informalidad es un fenómeno relativamente estable en el mercado de trabajo uruguayo, es decir, aparece como un rasgo de carácter estructural que no se ve afectado significativamente por los ciclos económicos (gráfica 1).

Gráfica 1. Crecimiento económico, informalidad y desempleo

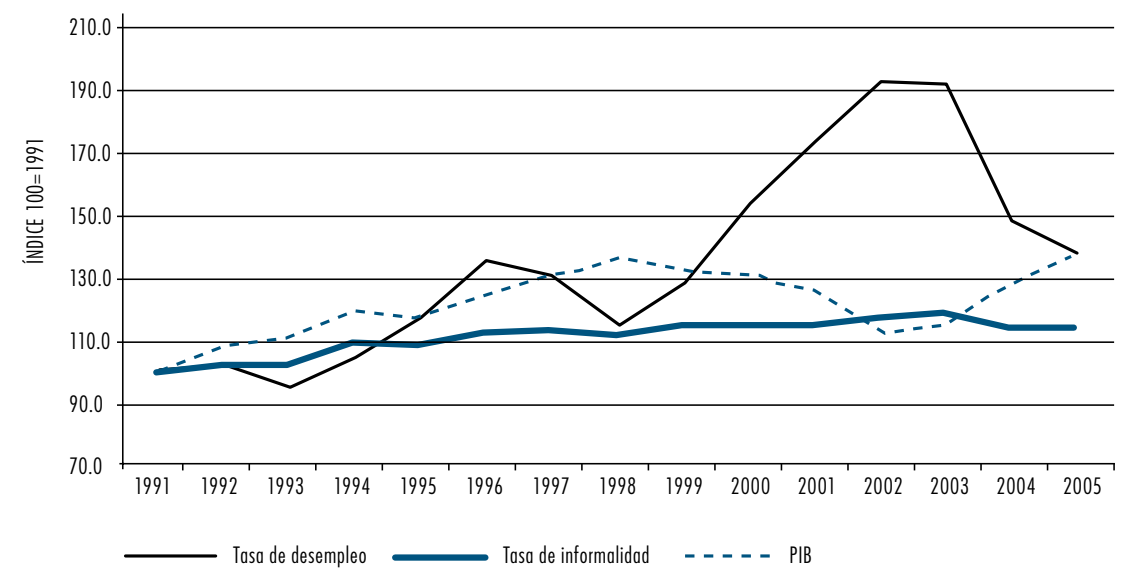

Fuente: elaborado con base en ECH, INE.

Si se analiza la información correspondiente a Uruguay durante las dos últimas décadas, se observa que entre 1991 y 1995 se verifica un crecimiento moderado de la informalidad -atribuible al comportamiento de la fuerza de trabajo masculina-, y luego se mantiene constante. La tasa de informalidad masculina se incrementó notablemente entre 1991 y 2005 , de $29 \%$ a $36.6 \%$, mientras que la femenina se mantuvo estable, $38.5 \%$ en 1991, 38.8\% en 2005. Estas tendencias, que dan lugar a una disminución en

5 Buenas revisiones sobre las aportaciones de estos trabajos pueden encontrarse en Longhi, 1998; Notaro, 2005; y Bucheli et al., 2002. 
la brecha de informalidad por sexo, coinciden con lo que ha ocurrido en el resto de los países de América Latina en los últimos 15 años (Abramo y Valenzuela, 2006).

El análisis por categoría de ocupación revela que la incidencia del empleo informal en 2005 abarca a $26.6 \%$ de los asalariados privados, $27.7 \%$ de los patrones o miembros de cooperativas, mientras que entre los trabajadores por cuenta propia sin local alcanza $94.4 \%$ (en esta categoría solamente se excluyen los profesionales) y $64.5 \%$ entre los trabajadores por cuenta propia con local. Por definición, el total de los trabajadores domésticos se considera dentro de la categoría informal. La incidencia de la informalidad resulta similar entre hombres y mujeres.

Si se combina el análisis de estas incidencias específicas con el peso de cada grupo en el total de ocupados, se visualiza que el grupo más importante dentro de los trabajadores informales en 2005 es el de los asalariados del sector privado (34\%), cuya participación relativa se ha incrementado en el periodo. Los trabajadores por cuenta propia sin local aumentaron su participación en el auge de la crisis económica (18.2\% en 1991; 24.6\% en 2002), expandiéndose en términos absolutos. A pesar de la incidencia similar de la informalidad entre hombres y mujeres, la composición del empleo informal difiere por sexo, debido a las diferencias en la inserción laboral de hombres y mujeres por categoría ocupacional. Entre los hombres, los asalariados privados y los trabajadores por cuenta propia tienen un peso relativo similar (37.4\% y $30.8 \%$, respectivamente). Entre las mujeres, la categoría principal corresponde a las trabajadoras domésticas (36\% del trabajo femenino informal), seguida por los asalariados privados (30.7 por ciento) $)^{6}$.

\section{Evolución de la desprotección social}

La desprotección social, entendida como la falta de cumplimiento de las normas vigentes sobre beneficios sociales, resulta una dimensión relevante del mercado laboral. Implica situaciones de desventaja relativa tanto en el presente -ausencia de prestaciones activas a los trabajadores- como en el futuro, debido a que los trabajadores no generan derechos para acceder a las prestaciones pasivas contributivas.

El principal componente del sistema de seguridad social en el país es el de las pasividades, que contempla la percepción de algunos beneficios durante la vida activa, entre otros, la cobertura médica a través de la Dirección de Seguros Sociales por Enfermedad (Disse).

6 Las mujeres representan $45 \%$ del total de trabajadores informales en 2005. 
El análisis de la cobertura de la seguridad social de los trabajadores uruguayos desde una perspectiva de largo plazo solamente puede realizarse de manera indirecta, a través de la información sobre cobertura de salud que surge de la ECH, la que indagaba si los individuos tenían derechos vigentes en alguna institución para atender su salud o si disponen de algún otro tipo de afiliación colectiva. A partir de 2001 se pregunta específicamente si el trabajador tiene derecho al pago de la afiliación a una institución de salud a través de la Disse y, de no tenerlo, se le pregunta si recibe algún pago en su empleo para este fin, además de los derechos jubilatorios. Estos cambios determinan un quiebre en la consistencia temporal de la serie que puede construirse a través de esta variable indirecta, como se señala en Bucheli (2004).

Utilizando esta variable, se definen como contribuyentes todos los asalariados públicos más los trabajadores del sector privado con derechos de atención de salud en el sistema mutual a través de la Disse o alguna forma de afiliación colectiva. La proxy de desprotección social así construida presenta una leve tendencia al incremento en el periodo analizado, resultado similar al señalado en Bucheli (2004). Los trabajadores no cubiertos pasan de $40.6 \%$ a $43.2 \%$ del total entre 1996 y 2001. Al igual que lo detectado en la informalidad, hay una disminución en la brecha entre hombres y mujeres, que se explica fundamentalmente por el incremento en la desprotección masculina.

A partir de 2001, el análisis de la información revela que entre ese año y 2004, el porcentaje de trabajadores sin derecho a jubilación pasa de $36 \%$ a $40 \%$, mientras que en 2005 se detecta una leve disminución. Esta última coincide con la etapa expansiva de la economía, el retorno a las convocatorias a Consejos de Salarios (organismos tripartitos de negociación salarial), la agudización de los controles para disminuir la evasión fiscal y las campañas publicitarias orientadas a disminuir la falta de registro a la seguridad social de los trabajadores.

El análisis por categoría de ocupación en 2005 muestra que los trabajadores por cuenta propia sin local presentan las mayores tasas de desprotección (94.7\%), seguidos por los trabajadores no remunerados (84\%), los trabajadores por cuenta propia con local (69\%) y los asalariados privados (34\%). No se advierten diferencias considerables entre hombres y mujeres, aunque el porcentaje de trabajadores sin derecho a jubilación entre las mujeres es superior debido a la concentración en el servicio doméstico, que se incluye dentro de las asalariadas privadas (37.6 por ciento).

Las tasas de incidencia presentadas deben analizarse junto con la composición del trabajo desprotegido. Se detecta así que aproximadamente la mitad de los trabajadores que no están amparados por la seguridad social son asalariados privados (41.5\%), y el peso de esta categoría es mayor entre las mujeres (55.6\%). Los trabajadores por cuenta propia, tanto con local como sin local también tienen un peso considerable ( $27 \%$ y $20 \%$, respectivamente). 
La desprotección social en 2005 es considerablemente superior entre los trabajadores del sector informal (74\%) que entre los del sector formal (21.5\%). El análisis de su evolución en el periodo refleja que el aumento de la desprotección (7.8\% entre 2001 y 2005) se registra para todos los trabajadores, e incluso es superior el aumento entre los formales (16.2\% frente a 7.8\% de variación entre 2001-2005). La caída de la desprotección social del último año obedece fundamentalmente a lo sucedido entre los trabajadores formales (variación negativa de 12.6 por ciento).

\section{¿La informalidad y la desprotección social afectan al mismo grupo de trabajadores?}

La respuesta a esta pregunta se ha ensayado para diversos países de América Latina (Bertranou, 2004), y se ha confirmado un alto grado de desprotección en el grupo

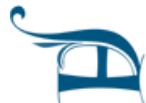
de trabajadores informales en todos los países de la región. La formalidad laboral no garantiza protección, aunque se asocia a una mayor probabilidad de contar con un conjunto de beneficios laborales y sociales.

Con el objetivo de analizar la superposición entre informalidad y desprotección social, se define operativamente como trabajadores vulnerables a la intersección de ambos grupos, situación que da cuenta de la desventaja relativa de estos trabajadores. Las personas o colectivos en esta situación son proclives a enfrentar mayores dificultades para el cuidado de su salud, solventar su vida como inactivos, eludir la pobreza y lograr estabilidad en su vida laboral ${ }^{7}$. El análisis se realiza a partir de 2001, ya que desde entonces la ECH permite detectar con rigurosidad a los trabajadores sin derecho a jubilación.

La superposición entre ambas problemáticas resulta clara: en 2005, 71.8\% de los trabajadores sin protección social son informales, mientras que la informalidad entre los trabajadores con protección social asciende a $15.9 \%$. Por el lado de la informalidad, $74 \%$ de los trabajadores informales no tienen protección social, mientras que entre los formales la desprotección social alcanza a 17.5 por ciento.

Más de una cuarta parte de los trabajadores $(27.8 \%)$ presenta ambas problemáticas: son informales y carecen de seguridad social. Sin embargo, a pesar de la alta superposición, ambas categorías no se solapan por completo, sino que existe desprotección social entre los trabajadores formales, y a su vez algunos trabajadores con protección social se insertan en lo que tradicionalmente se ha denominado el sector informal.

7 Se trata de una definición operativa para facilitar la clasificación. Debe señalarse que el mismo término ha sido utilizado, por ejemplo, por Bertranou y Gasparini (2005), para describir la situación de los empleados y desempleados. 


\section{Características de los trabajadores}

Con el objetivo de determinar el efecto independiente de las características personales y del hogar en la probabilidad de estar en situación de informalidad, desprotección social y vulnerabilidad, se realizó un análisis multivariado basado en la estimación de modelos de variable binaria dependiente (probit). Se estimaron tres modelos básicos, donde la variable dependiente indica, alternativamente, si el trabajador es informal, si no aporta a la seguridad social o si es vulnerable. Para cada uno de estos modelos se estimaron dos alternativas, siguiendo el ejercicio presentado en CEPAL (2006). La primera de ellas (modelo 1) considera como variables explicativas a las que reflejan las características no laborales de los individuos, es decir, aquéllas vinculadas a aspectos personales o asociadas a sus hogares y lugar de residencia. La segunda alternativa (modelo 2) agrega las variables relacionadas con la inserción laboral del individuo, o sea que en este caso se consideran las características de los puestos de trabajo. En cuanto a la informalidad, se agregan las ramas de actividad; y respecto de la falta de aportaciones, se incluyen controles para algunos tipos de ocupación y categorías ocupacionales. Las variables explicativas incluidas en los distintos modelos se presentan en el cuadro A-1 (véase anexo) y los resultados de las estimaciones en el cuadro 1.

Los resultados indican que la probabilidad de ser informal o vulnerable es menor para las mujeres, pero esta variable pierde significación para los modelos de no aportar. Resulta interesante destacar que cuando no se incluyen los controles por rama de actividad, el sexo no resulta significativo ni en el modelo de informalidad ni en el de vulnerabilidad. Esto indica que, al interior de una rama de actividad, las mujeres tienen una menor probabilidad de ser informales o vulnerables.

Las edades centrales ( 25 a 34 años) tienen menor probabilidad de ser informales o vulnerables, mientras que los grupos en edades extremas tienen mayor probabilidad de estar en situación de informalidad o vulnerabilidad. Se detecta un patrón diferente en el caso de las contribuciones a la seguridad social, con probabilidades de no aportar decrecientes con el tramo etario, y una mayor probabilidad de no aportar de los más jóvenes. Los trabajadores de mayor edad tienen más probabilidad de aportar que el grupo omitido, lo que se relaciona con la proximidad del momento de retiro. En los tres modelos, los trabajadores más jóvenes se configuran como un grupo en situación desventajosa. Todos los resultados relativos a la edad se mantienen al introducir los controles por rama de actividad.

Respecto de la relación de parentesco, la variable que distingue a los cónyuges solamente es significativa en el caso del modelo de cotización a la seguridad social en sus dos especificaciones, indicando una mayor probabilidad de que estos trabajadores 
Cuadro 1. Probabilidad de ser informal, de no aportar a la seguridad social o ser vulnerable (2005)

\begin{tabular}{|c|c|c|c|c|c|c|}
\hline & \multicolumn{2}{|c|}{ Informalidad } & \multicolumn{2}{|c|}{ No aportar } & \multicolumn{2}{|c|}{ Vulnerabilidad } \\
\hline & Modelo (1) & Modelo (2) & Modelo (1) & Modelo (2) & Modelo (1) & Modelo (2) \\
\hline Mujer & $0.083(3.65)^{\star \star \star}$ & $-0.062(2.25)^{\star \star}$ & $-0.012(0.43)$ & $-0.030(1.01)$ & 0.040 (1.63) & $-0.073(2.48)^{\star \star}$ \\
\hline 14 a 17 & $0.219(2.63)^{\star \star \star}$ & $0.070(0.78)$ & $1.695(9.94)^{\star \star \star}$ & $1.709(9.85)^{\star \star \star}$ & $0.389(4.57)^{\star \star \star}$ & $0.356(3.99)^{\star \star \star *}$ \\
\hline 18 a 24 & $0.065(1.88)^{*}$ & $0.004(0.11)$ & $0.302(7.62)^{\star \star \star}$ & $0.307(7.65)^{\star \star \star}$ & $0.124(3.32)^{\star \star \star}$ & $0.098(2.47)^{\star \star}$ \\
\hline 35 a 44 & $0.070(2.43)^{\star \star}$ & $0.111(3.43)^{\star \star \star}$ & $-0.183(5.26)^{\star \star \star}$ & $-0.176(5.02)^{\star \star \star *}$ & $0.022(0.69)$ & $0.052(1.53)$ \\
\hline 45 a 64 & $0.123(4.37)^{\star \star \star}$ & $0.199(6.22)^{\star \star \star}$ & $-0.188(5.49)^{\star \star \star}$ & $-0.187(5.37)^{\star \star \star}$ & $0.080(2.60)^{\star * \star}$ & $0.136(4.07)^{* \star *}$ \\
\hline 65 y más & $0.374(6.60)^{\star \star \star}$ & $0.467(7.25)^{\star \star \star}$ & $-0.172(2.52)^{\star \star}$ & $-0.175(2.52)^{\star \star}$ & $0.269(4.53)^{\star \star \star}$ & $0.337(5.37)^{\star \star \star}$ \\
\hline Cónyuge & $-0.021(0.72)$ & $-0.031(0.92)$ & $0.090(2.58)^{\star \star \star *}$ & $0.099(2.81)^{\star \star \star}$ & $0.019(0.61)$ & $0.001(0.02)$ \\
\hline Hiijo & $0.129(4.18)^{\star \star \star}$ & $0.109(3.19)^{\star \star \star}$ & $0.333(9.07)^{\star \star \star}$ & $0.334(8.99)^{\star \star \star}$ & $0.220(6.61)^{\star \star \star}$ & $0.196(5.49)^{\star \star \star *}$ \\
\hline Otro pariente & $0.179(4.37)^{\star \star \star}$ & 0.057 (1.09) & $0.396(8.21)^{\star \star \star}$ & $0.389(8.00)^{\star \star \star}$ & $0.248(5.67)^{\star \star \star}$ & $0.168(3.59)^{\star \star \star *}$ \\
\hline Región & $-0.202(10.3)^{\star \star \star}$ & $-0.240(10.7)^{\star \star \star}$ & $-0.133(5.54)^{\star \star \star}$ & $-0.145(5.89)^{\star \star \star \star}$ & $-0.206(9.70)^{\star \star \star \star}$ & $-0.252(10.9)^{\star \star *}$ \\
\hline Cobra jubilación & $0.215(3.94)^{\star \star \star}$ & 0.075 (1.24) & $0.623(9.51)^{\star \star \star *}$ & $0.629(9.43)^{\star \star \star}$ & $0.389(6.85)^{\star \star \star}$ & $0.293(4.86)^{\star \star \star}$ \\
\hline Otros ingresos & $0.006(0.59)$ & 0.014 (1.25) & 0.040 (1.52) & $0.043(1.69)^{*}$ & $0.092(2.21)^{* *}$ & $0.060(1.88)^{\star}$ \\
\hline Otros ingresos 2 & $-0.016(8.79)^{\star \star \star}$ & $-0.014(6.90)^{\star \star \star}$ & $-0.033(7.94)^{\star \star \star}$ & $-0.032(7.92)^{\star \star \star}$ & $-0.044(6.50)^{\star \star \star}$ & $-0.035(6.63)^{\star \star \star \star}$ \\
\hline T. dependencia & $-0.449(7.47)^{\star \star \star *}$ & $-0.413(6.18)^{\star \star \star}$ & $-0.442(5.99)^{\star \star \star \star}$ & $-0.424(5.69)^{\star \star \star}$ & $-0.504(7.58)^{\star \star \star}$ & $-0.446(6.25)^{\star \star * \star}$ \\
\hline Otros aportantes & $-0.054(3.78)^{\star \star \star}$ & $-0.061(3.87)^{\star \star \star}$ & $-0.207(12.2)^{\star \star \star}$ & $-0.205(12.0)^{\star \star \star \star}$ & $-0.142(8.97)^{\star \star \star \star}$ & $-0.154(9.08)^{\star \star \star *}$ \\
\hline Asiste & $-0.139(3.18)^{\star \star \star *}$ & $-0.130(2.73)^{\star \star \star}$ & $0.140(2.70)^{\star \star \star *}$ & $0.144(2.75)^{\star \star \star}$ & $-0.064(1.32)$ & $-0.073(1.42)$ \\
\hline Sec. ler ciclo & $-0.267(10.5)^{\star \star \star}$ & $-0.133(4.57)^{\star \star \star}$ & $-0.258(8.35)^{\star \star \star}$ & $-0.268(8.50)^{\star \star \star}$ & $-0.292(10.9)^{\star \star \star}$ & $-0.206(7.16)^{\star \star \star *}$ \\
\hline Sec. 20 ciclo & $-0.584(16.2)^{\star \star \star}$ & $-0.285(6.99)^{\star \star \star}$ & $-0.519(11.6)^{\star \star \star}$ & $-0.489(10.7)^{\star \star \star}$ & $-0.676(16.6)^{\star \star \star \star}$ & $-0.446(10.1)^{\star \star \star \star}$ \\
\hline Técnica & $-0.254(8.38)^{\star \star * \star}$ & $-0.005(0.14)$ & $-0.353(9.39)^{\star \star *}$ & $-0.368(9.60)^{\star \star \star *}$ & $-0.310(9.63)^{\star \star \star}$ & $-0.164(4.69)^{\star \star \star \star}$ \\
\hline Maestro/Prof. & $-1.688(25.6)^{\star \star \star}$ & $-0.533(5.85)^{\star \star \star *}$ & $-0.682(7.83)^{\star \star \star}$ & $-0.667(7.26)^{\star \star \star}$ & $-1.610(21.6)^{\star \star \star}$ & $-0.519(5.20)^{\star \star \star *}$ \\
\hline Univ. Inc. & $-0.796(17.8)^{\star \star \star}$ & $-0.353(7.12)^{\star \star \star}$ & $-0.616(11.2)^{\star \star \star}$ & $-0.533(9.55)^{\star \star \star}$ & $-0.940(17.8)^{\star \star \star}$ & $-0.567(10.0)^{\star \star * *}$ \\
\hline Univ. Comp. & $-1.556(29.6)^{\star \star \star}$ & $-1.042(17.7)^{\star \star \star}$ & $-1.187(18.7)^{\star \star \star}$ & $-1.003(15.2)^{\star \star \star \star}$ & $-1.666(23.3)^{\star \star \star}$ & $-1.206(15.5)^{\star \star \star \star}$ \\
\hline Tiempo comp. & $-0.753(35.4)^{\star \star \star}$ & $-0.830(33.4)^{\star \star \star *}$ & $-0.762(28.5)^{\star \star \star}$ & $-0.775(27.9)^{\star \star \star}$ & $-0.952(42.0)^{\star \star \star \star}$ & $-0.993(39.9)^{\star \star \star *}$ \\
\hline Doble empleo & $-0.130(3.78)^{\star \star *}$ & $-0.136(3.28)^{\star \star \star}$ & $0.001(0.02)$ & $0.001(0.02)$ & $-0.175(4.58)^{\star \star \star \star}$ & $-0.199(4.65)^{\star \star *}$ \\
\hline Serv. doméstico & & & $0.116(2.39)^{\star \star \star}$ & $-0.319(4.26)^{\star \star \star}$ & & \\
\hline Func. Público & & & $-1.197(18.88)^{\star \star \star \star}$ & $-1.114(12.40)^{\star \star \star}$ & & \\
\hline Directivo & & & $-0.392(10.4)^{\star * \star *}$ & $-0.371(9.34)^{\star \star \star}$ & & \\
\hline Cuenta propia & & & $0.907(27.2)^{\star \star \star}$ & $0.943(26.8)^{\star \star \star}$ & & \\
\hline Microemp. & & & $0.953(31.9)^{\star \star \star}$ & $0.904(28.3)^{\star \star \star}$ & & \\
\hline Agricultura, etc. & & $0.347(7.01)^{\star \star \star}$ & & $-0.377(6.72)^{\star \star \star *}$ & & $-0.302(5.55)^{\star \star \star *}$ \\
\hline Electricidad & & $-2.164(5.76)^{\star \star \star \star}$ & & $-0.355(1.19)$ & & $-1.952(5.09)^{\star \star \star *}$ \\
\hline Construcción & & $0.590(13.3)^{\star \star \star}$ & & $0.164(3.08)^{\star \star \star}$ & & $0.581(12.8)^{\star \star \star *}$ \\
\hline Comercio, hoteles y restaurantes & & $0.130(4.20)^{\star \star \star}$ & & $-0.154(4.18)^{\star \star \star}$ & & $-0.022(0.66)$ \\
\hline Transporte, etc. & & $0.017(0.37)$ & & $-0.463(8.08)^{\star \star \star}$ & & $-0.379(6.96)^{\star \star \star \star}$ \\
\hline Inst. financieras y afines & & $-0.016(0.39)$ & & $-0.482(9.99)^{\star \star \star \star}$ & & $-0.226(5.00)^{\star * *}$ \\
\hline Admón. púb. y defensa & & $-2.588(15.3)^{\star \star \star *}$ & & $-0.312(2.56)^{\star \star}$ & & \\
\hline Enseñanza & & $-1.835(18.7)^{\star \star \star}$ & & $-0.233(2.86)^{\star \star \star}$ & & $-1.992(15.9)^{\star \star *}$ \\
\hline Servicios soc. y salud & & $-0.734(14.1)^{\star \star \star *}$ & & $-0.432(7.22)^{\star \star \star}$ & & $-0.722(12.3)^{\star \star \star \star}$ \\
\hline Servicios comun., soc. y pers. & & $-0.031(0.68)$ & & $0.151(2.78)^{\star \star \star}$ & & $-0.096(1.98)^{\star \star}$ \\
\hline Hogares con servicio doméstico & & $2.366(29.4)^{\star \star \star}$ & & $0.342(4.61)^{\star \star \star *}$ & & $0.809(18.2)^{\star \star *}$ \\
\hline Org. extraterritorial & & & & $0.990(2.66)^{\star \star \star}$ & & \\
\hline Constante & $0.766(18.2)^{\star \star \star}$ & $0.600(11.3)^{\star \star \star}$ & $0.346(5.4)^{\star \star \star *}$ & $0.487(7.09)^{\star \star \star}$ & $0.678(9.42)^{\star \star \star}$ & $0.732(10.6)^{\star \star *}$ \\
\hline Observaciones & 22123 & 22106 & 22123 & 22122 & 22123 & 20364 \\
\hline Count R2: & 0.780 & 0.722 & 0.848 & 0.843 & 0.803 & 0.792 \\
\hline
\end{tabular}


sean no aportantes en relación con el jefe del hogar. Las variables que distinguen a los hijos y a los otros parientes son significativas en todos los modelos (a excepción de los otros parientes en el modelo 2 de informalidad) y el signo es positivo, indicando una mayor dificultad de inserción que los jefes del hogar.

Para las tres problemáticas consideradas, el efecto de la región presenta signo negativo, dando cuenta de la menor probabilidad de estar en estas situaciones para los trabajadores de Montevideo.

Como era previsible, los trabajadores que son perceptores de jubilaciones tienen una mayor probabilidad de no aportar a la seguridad social. Es importante recordar que la combinación de empleo y percepción de jubilaciones no siempre representa una ilegalidad, ya que los trabajadores pueden estar percibiendo jubilaciones por una caja y realizando aportaciones a otra. En el caso de la informalidad, quienes reciben jubilación tienen una mayor probabilidad de ser informales, aunque el efecto deja de ser significativo al introducir los controles por rama de actividad. Sin embargo, esta asociación positiva entre recibir jubilación y ser no aportante es la que prevalece en el caso de la vulnerabilidad.

El ingreso per cápita del resto del hogar (en logaritmos) presenta una significación débil en el modelo de no aportar (modelo 2) y en el de vulnerabilidad, en ambos casos con signo positivo. Esto indica que los trabajadores que viven en hogares donde el resto de los miembros tienen bajos ingresos, presentan una mayor probabilidad de realizar contribuciones, lo que podría asociarse a que son los principales perceptores de ingresos. En todos los casos la expresión cuadrática de esta variable presenta signo negativo.

La proporción de personas dependientes en el hogar, medida como el número de inactivos no perceptores de ingresos sobre el total de personas en el hogar, presenta signo negativo y resulta significativa en todos los casos. Este resultado, que también se ha encontrado en trabajos previos, ha sido interpretado sugiriendo que dado el nivel educativo la necesidad de sostener a un mayor número de personas aumenta la probabilidad de contribuir a la seguridad social (Bucheli, 2004).

La presencia de otros trabajadores que aporten a la seguridad social en el hogar disminuye la probabilidad de ser un trabajador informal, no cotizante o vulnerable. Este resultado indicaría la tendencia a la formación de hogares relativamente homogéneos en cuanto a la calidad de la inserción laboral, marcando una tendencia a la polarización de este tipo de problemas. Los individuos tienden a agruparse en hogares formados por trabajadores con características laborales similares, por ejemplo, hogares de empleos "de baja calidad".

Los individuos que asisten al sistema educativo tienen menor probabilidad de ser informales, pero mayor de no aportar a la seguridad social. Esto último podría indicar una 
estrategia de los jóvenes para evadir las aportaciones en esta etapa de su ciclo de vida. Los efectos contradictorios determinan que la variable no resulte significativa para los trabajadores vulnerables. A medida que aumenta el nivel educativo de los trabajadores, disminuye la probabilidad de que sean informales, no aportantes a la seguridad social o vulnerables. Se trata de un efecto robusto que se repite en los distintos modelos.

Los trabajadores de tiempo completo tienen menor probabilidad de ser informales, de no aportar a la seguridad social o ser vulnerables. Esto señala una probable superposición de las malas condiciones de trabajo en los mismos individuos, ya sean trabajadores formales o informales, que no aportan a la seguridad social que se relacionan con insuficiencia de horas trabajadas. En el caso de los trabajadores con más de un empleo, se detecta una asociación negativa a la probabilidad de ser informal o vulnerable, pero la variable no resulta significativa en relación con la probabilidad de no cotizar a la seguridad social. Sobre este último resultado, cabe destacar que los mayores niveles de multiempleo tienen lugar entre los trabajadores del sector público (enseñanza, salud), y el modelo de no cotizantes incluye una variable que distingue a los funcionarios públicos, que sí resulta significativa y con signo negativo.

Los controles por rama de actividad fueron incluidos considerando a la industria como sector de comparación. Los trabajadores del sector primario de la economía (agricultura, ganadería, pesca y minería) presentan una mayor probabilidad de ser informales que los trabajadores de la industria, lo que se relaciona con el tamaño de los establecimientos. Sin embargo, la probabilidad de los trabajadores de esta rama de no aportar a la seguridad social es menor en relación con la industria, y éste es el efecto que prevalece entre los vulnerables. Los trabajadores de electricidad, gas y agua tienen menor probabilidad de ser informales y vulnerables, debido al predominio de empresas públicas en estas actividades. La rama pierde significación en el modelo de aportaciones a la seguridad social, debido a que se trata de una rama típicamente de asalariados públicos, y este modelo incluye una variable que los distingue.

Los trabajadores de la construcción presentan una mayor probabilidad de ser informales, no aportantes o vulnerables en comparación con la industria, señalando las condiciones relativas desventajosas de esta rama de actividad. El comercio, los hoteles y los restaurantes presentan mayor probabilidad de ser informal, y menor respecto a no contribuir a la seguridad social, mientras que en el caso de la vulnerabilidad no presenta significación. Estos resultados se explican debido a la alta participación de pequeñas empresas que al mismo tiempo presentan relativamente una cobertura de la seguridad social considerable entre sus trabajadores. Por su parte, el transporte presenta efectos no significativos en cuanto a la informalidad y menor probabilidad respecto a no aportar o ser trabajador vulnerable. 
Como era de esperarse, el conjunto de actividades de intermediación financiera, servicios inmobiliarios y a empresas tiene una menor probabilidad que la industria de no aportar o ser vulnerable, aunque no es significativo respecto a la informalidad. La inserción laboral en la administración pública, enseñanza y salud se asocia a menores probabilidades de ser informales o no aportar, ya que estas ramas concentran a los funcionarios públicos. Los servicios comunitarios, personales, etc. presentan una mayor probabilidad de no aportar y de ser vulnerable pero no muestran significación para la informalidad.

Interesa en especial analizar lo que acontece con la rama de servicio doméstico en hogares privados, que se asocia a una mayor probabilidad de falta de aportaciones a la seguridad social o de inserción informal. En relación con la falta de aportaciones, esta variable debe interpretarse conjuntamente con la variable binaria que indica si el trabajador pertenece al servicio doméstico. Si se distingue al servicio doméstico del resto de las trabajadoras de la rama, las primeras tienen mayor probabilidad de contribuir, ya que el resto de las ocupaciones femeninas de la rama 95 (CIIU, Rev. 3) presentan peores condiciones que las trabajadoras del servicio doméstico. Esto nos conduce a preguntarnos en qué medida se logra captar la especificidad del trabajo del servicio doméstico considerando solamente la ocupación 913. En el caso de las mujeres, se excluye a las niñeras, cuidadoras de enfermos, etc. (que se incluyen en las ocupaciones 513 y 514), cuyas tareas y relación con los empleadores podrían ser asimilables al servicio doméstico.

Como era previsible, los funcionarios públicos y directivos tienen menor probabilidad de no realizar aportaciones a la seguridad social; lo contrario ocurre entre los trabajadores por cuenta propia y los microempresarios.

\section{Industria}

La estructura relativa de remuneraciones pone de manifiesto la heterogeneidad al interior de los trabajadores clasificados como informales. Su remuneración promedio en 2005 equivale a 55\% del promedio correspondiente al total de ocupados. Los trabajadores independientes sin local y, en particular, los trabajadores del servicio doméstico registran las remuneraciones más bajas en términos relativos, especialmente cuando se consideran las remuneraciones mensuales.

El servicio doméstico deja de ser el grupo más rezagado cuando se analizan las remuneraciones horarias, y este lugar pasa a ser ocupado por los asalariados privados informales y trabajadores por cuenta propia sin local. Los patrones y cooperativistas informales reciben remuneraciones promedio superiores al total de ocupados (casi $90 \%$ 
mayores en 2005), aunque la brecha disminuye levemente al considerar las remuneraciones horarias. Los trabajadores por cuenta propia con local constituyen un grupo intermedio, con remuneraciones mensuales equivalentes a $69 \%$ de la remuneración total promedio en 2005 , brecha que se reduce al considerar las remuneraciones horarias.

La falta de cobertura de la seguridad social está fuertemente asociada a remuneraciones relativas menores (63\% del total en el caso de las remuneraciones mensuales, $73 \%$ si consideramos las horarias). El grupo de trabajadores vulnerables se configura como el peor posicionado, con remuneraciones mensuales equivalentes a alrededor de $38 \%$ del promedio de la economía en 2005, y 52\% si se consideran las remuneraciones horarias. Sobre estos diferenciales es probable que actúen de manera conjunta aspectos antes reseñados, como los bajos niveles educativos y la menor cantidad de horas trabajadas.

Las diferencias salariales que surgen de esta simple comparación de promedios podrían estar afectadas por las características personales de los trabajadores. Para controlar de acuerdo con estas características, se estimaron ecuaciones de Mincer, utilizando como variable dependiente las remuneraciones horarias y las mensuales. Se distinguen cuatro grupos de trabajadores: vulnerables, formales que aportan, formales que no aportan e informales que aportan. Estos cuatro grupos se identifican por medio de variables binarias. Tanto para el total de trabajadores como para hombres y mujeres, las mayores remuneraciones corresponden al grupo de trabajadores formales y que aportan a la seguridad social. En segundo lugar se ubican los informales que aportan, luego los formales que no aportan y finalmente los trabajadores vulnerables (categoría excluida en la regresión) (cuadro 2). El ordenamiento de los grupos que surge de las ecuaciones con remuneraciones mensuales y horarias es similar. Estos resultados indican que la condición de aportar o no a la seguridad social es la determinante en el ordenamiento de los trabajadores de acuerdo con sus ingresos.

Para ilustrar las diferencias salariales, se estimaron funciones de densidad kernel. Los trabajadores formales que aportan se sitúan en el extremo derecho de la distribución, y las curvas correspondientes a los que no aportan, formales e informales, tienden a superponerse, reflejando su similitud. Ambas se sitúan en el extremo izquierdo de la distribución. La densidad correspondiente a los vulnerables, es decir, que no aportan y son informales, es la que se ubica más hacia la izquierda (gráfica 2). 
Gráfica 2. Densidad de las remuneraciones (2005)

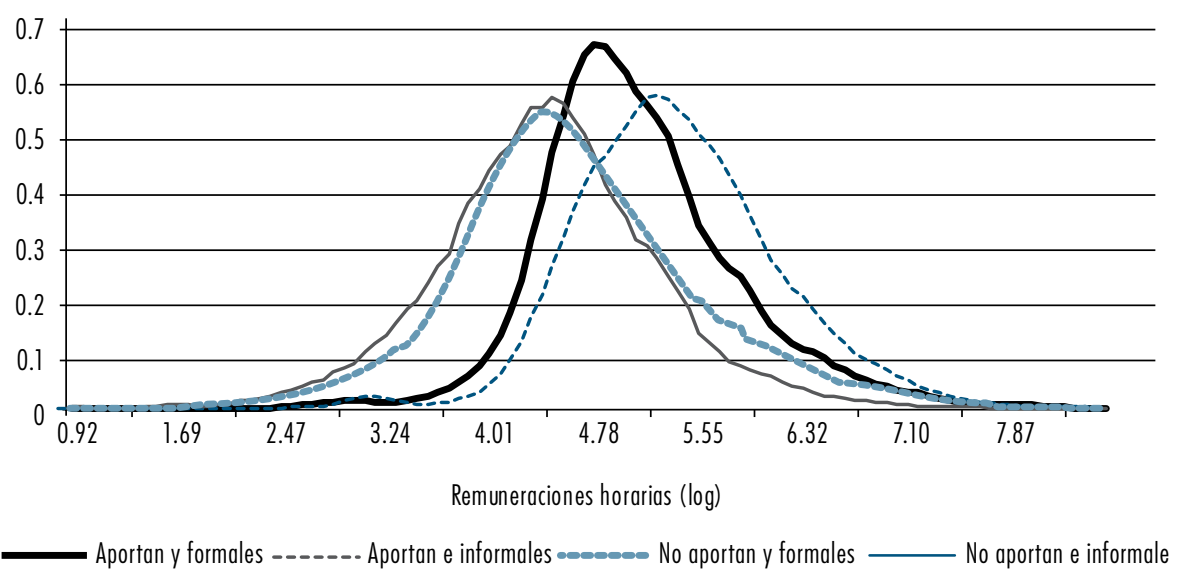

Fuente: elaborado con base en ECH, INE.

\section{Comentarios finales}

En Uruguay, al igual que en el ámbito internacional, existe una relación directa entre informalidad y desprotección social, en cuanto esta última es una de las características sobresalientes del empleo informal. La informalidad, entendida en la concepción tradicional de la OIT, es un fenómeno relativamente estable en la esfera nacional, que no registra cambios significativos a lo largo de los ciclos económicos. Se detecta una tendencia al incremento de la desprotección social en los últimos años, aunque en 2005 éste se reduce. El análisis de las problemáticas de informalidad y desprotección señala que, a pesar de que existe una alta superposición, no es total. Dentro de la categoría informal se engloban una variedad de situaciones y gran heterogeneidad, lo que lleva a cuestionarse sobre la pertinencia de ese concepto para el estudio de la calidad del empleo en Uruguay. Tales cuestionamientos ya fueron planteados en trabajos anteriores (Notaro, 2005), y son reforzados por la evidencia presentada en este documento. Cuando se considera la situación relativa de los trabajadores en función de sus remuneraciones, los mejor posicionados son los trabajadores formales que aportan a la seguridad social. En segundo lugar se ubican los informales que aportan, luego los formales que no aportan y finalmente los trabajadores vulnerables. Estos resultados indican que la condición de aportar o no aportar a la seguridad social es la determinante en el ordenamiento de los trabajadores de acuerdo con sus ingresos, y fortalecen la idea de que la categoría de desprotección social resulta más adecuada para el estudio de la calidad del trabajo en Uruguay. Sin embargo, se hace necesario 
Cuadro 2. Ecuaciones salariales (2005)

Variable dependiente: remuneraciones $(\log )$

\begin{tabular}{|c|c|c|c|c|c|c|}
\hline \multicolumn{5}{|c|}{ Remuneraciones horarias } & \multicolumn{2}{|c|}{ Remuneraciones mensuales } \\
\hline & Todos & Hombres & Mujeres & Todos & Hombres & Mujeres \\
\hline Sexo & $-0.187(17.28)^{\star \star \star}$ & & & $-0.350(30.67)^{\star \star \star}$ & & \\
\hline Edad & $0.013(32.03)^{\star * *}$ & $\star 0.015(28.69)^{\star \star \star}$ & $0.009(15.13)^{\star \star \star}$ & $0.013(30.41)^{\star \star * \star}$ & $\star 0.015(26.99)^{\star \star *}$ & $0.010(15.04)^{\star \star \star}$ \\
\hline Región & $0.201(20.39)^{* * *}$ & $\star 0.184(13.76)^{\star \star \star \star}$ & $0.228(15.66)^{* * *}$ & $0.150(14.25)^{\star \star \star}$ & $\star 0.127(9.01)^{\star \star \star}$ & $0.179(11.43)^{\star * *}$ \\
\hline $\begin{array}{l}\text { Secundaria } \\
\text { incompleta }\end{array}$ & $0.250(18.36)^{* * \star}$ & $\star 0.295(17.07)^{\star \star \star}$ & $0.170(7.73)^{\star \star \star}$ & $0.277(18.87)^{\star \star \star}$ & $\star 0.317(17.21)^{\star \star \star *}$ & $0.213(8.83)^{\star \star \star}$ \\
\hline $\begin{array}{l}\text { Secundaria } \\
\text { completa }\end{array}$ & $0.531(28.57)^{\star \star \star}$ & $\star 0.588(22.79)^{\star \star \star \star}$ & $0.439(15.90)^{* \star *}$ & $0.549(28.82)^{\star \star \star}$ & $\star 0.675(22.93)^{\star \star \star}$ & $0.454(16.17)^{\star \star \star x}$ \\
\hline Técnica & $0.359(21.85)^{\star \star \star}$ & $\star 0.378(18.58)^{\star \star \star \star}$ & $0.303(10.90)^{* \star *}$ & $0.355(20.03)^{\star * \star}$ & $\star 0.380(17.42)^{\star \star \star *}$ & $0.299(9.82)^{\star \star \star}$ \\
\hline $\begin{array}{l}\text { Magisterio/ } \\
\text { profesorado }\end{array}$ & $0.771(28.08)^{\star * *}$ & $\star 0.698(13.91)^{\star * \star}$ & $0.707(20.34)^{\star \star \star}$ & $0.626(21.08)^{\star \star \star \star}$ & $\star 0.537(8.95)^{\star \star \star}$ & $0.549(14.93)^{\star \star \star *}$ \\
\hline $\begin{array}{l}\text { Terciaria } \\
\text { incompleta }\end{array}$ & $0.717(35.44)^{\star \star *}$ & $\star 0.763(26.87)^{\star \star \star}$ & $0.609(20.42)^{\star * \star}$ & $0.610(28.76)^{\star \star \star *}$ & $\star 0.668(22.69)^{\star \star * \star}$ & $0.503(15.82)^{\star \star \star x}$ \\
\hline $\begin{array}{l}\text { Terciaria } \\
\text { completa }\end{array}$ & $1.105(51.92)^{\star \star *}$ & $\star 1.201(39.33)^{\star \star \star \star}$ & $0.964(31.16)^{\star \star *}$ & $0.995(46.63)^{\star \star \star *}$ & * $1.138(37.94)^{\star \star \star}$ & $0.821(26.01)^{\star \star \star *}$ \\
\hline Formal y aporta & $0.627(44.03)^{\star * \star}$ & $\star 0.623(34.74)^{\star \star \star \star}$ & $0.622(25.59)^{\star \star \star \star}$ & $1.151(72.66)^{\star \star \star}$ & * $1.082(54.34)^{\star \star \star}$ & $1.261(47.14)^{\star \star \star x}$ \\
\hline Formal y no aporta & $0.160(7.69)^{\star \star \star}$ & $0.154(5.97)^{\star \star \star}$ & $0.168(4.78)^{\star \star \star}$ & $0.411(17.95)^{\star \star \star}$ & $\star 0.366(12.85)^{\star \star \star *}$ & $0.491(12.80)^{\star \star * x}$ \\
\hline Informal y aporta & $0.524(29.84)^{\star * \star}$ & $\star 0.570(22.97)^{\star \star \star}$ & $0.464(18.84)^{\star \star \star}$ & $0.952(51.29)^{\star \star \star \star}$ & $\star 0.960(37.31)^{\star \star \star *}$ & $0.938(34.85)^{\star \star \star x}$ \\
\hline Constante & $4.413(31.14)^{\star \star \star}$ & $\star 3.905(83.04)^{\star \star \star}$ & $3.778(24.87)^{\star \star \star *}$ & $7.847(53.85)^{\star \star \star}$ & $\star 7.243(147.73)^{\star \star \star}$ & $6.674(60.00)^{\star * *}$ \\
\hline Controles por rama & Si & Si & Si & Si & Si & Si \\
\hline Observaciones & 21733 & 12087 & 9646 & 21733 & 12087 & 9646 \\
\hline$R-2$ & 0.42 & 0.43 & 0.44 & 0.50 & 0.47 & 0.51 \\
\hline
\end{tabular}

$*$ significativo al $10 \%$; $* *$ significativo al $5 \%$; *** significativo al $1 \%$

Fuente: elaborado con base en ECH, INE.

contar con un estudio más amplio sobre calidad del empleo, que abarque diferentes dimensiones y no solamente el ingreso, para tener mayor evidencia al respecto.

La desprotección asociada a la inserción como asalariados refleja una problemática diferente a la de los trabajadores por cuenta propia. En el primer caso parece remitir a un problema de costos laborales y evasión, en la medida en que la decisión sobre el registro de los trabajadores está, en última instancia, en manos del empleador. Para los trabajadores independientes, las causas de la desprotección parecen estar asociadas tanto a los bajos ingresos obtenidos como a la falta de adecuación de los sistemas de afiliación a la seguridad social a la realidad de estos trabajadores. En este caso no parece que pueda refrendarse la hipótesis de una respuesta racional de los agentes económicos. El origen de las diversas situaciones es heterogéneo y sugiere la necesidad de diferentes políticas 
públicas orientadas a diversos objetivos. Más allá de estos indicios, sería interesante avanzar en esta línea de investigación para determinar cuál de los enfoques planteados (dualista, legalista o estructuralista) se adapta mejor al caso uruguayo como un todo o a las diferentes situaciones detectadas.

El caso del servicio doméstico parece revestir connotaciones distintas, relacionadas con la falta de reconocimiento del valor económico del trabajo doméstico remunerado y no remunerado, y la desvalorización social de actividades consideradas típicamente femeninas. A ello se agregan las dificultades de fiscalización de una actividad que se desarrolla en hogares, así como la falta de organización de las trabajadoras por la forma individual y aislada en que se realizan estas actividades. Se trata de un segmento específico del mercado laboral femenino que requiere políticas especiales, máxime considerando el papel de las mujeres en la reproducción social.

\section{Bibliografía}

Abramo, Laís y María Elena Valenzuela, "Inserción laboral y brechas de equidad de género en América Latina", en Laís Abramo (ed.), Trabajo decente y equidad de género en América Latina, Santiago de Chile, Oficina Internacional del Trabajo, 2006, pp. 29-62.

Amarante, Verónica y Rodrigo Arim, "El mercado laboral: Cambios estructurales y el impacto de la crisis, 1986-2002", en Uruguay: empleo y protección social; de la crisis al crecimiento, Santiago de Chile, Oficina Internacional del Trabajo, 2005a, pp. 39-133.

"Acciones públicas a favor del empleo en Uruguay", en Algunos factores a tener en cuenta para la participación de las micro y pequeñas empresas en el desarrollo económico local y la generación de empleo decente en Uruguay, Programa Redel Uruguay, Documento de trabajo, Montevideo, Programa Redel, 2005b, pp. 51-61.

"Empleo informal en Uruguay", en Algunos factores a tener en cuenta para la participación de las micro y pequeñas empresas en el desarrollo económico local y la generación de empleo decente en Uruguay, Programa Redel Uruguay, Documento de trabajo, Montevideo, Programa Redel, 2005b, pp. 63-73.
Bertranou, Fabio, “¿Desarticulación o subordinación? Protección social y mercado laboral en América Latina", en Fabio Bertranou (ed.), Protección social y mercado laboral, Santiago de Chile, Oficina Internacional del Trabajo, 2004, pp. 13-28.

Bucheli, Marisa, Insumos para la elaboración de un proyecto sobre informalidad, Montevideo, IPES, Universidad Católica del Uruguay, 2002.

, "Uruguay: La cobertura de la seguridad social en el empleo, 1991-2002", en Fabio Bertranou (ed.), Protección social y mercado laboral, Santiago de Chile, Oficina Internacional del Trabajo, 2004, pp. 163-197.

Bulow Jeremy y Lawrence Summers, "A theory of dual labor markets with application to industrial policy, discrimination and keynesian unemployment", Journal of Labor Economics, vol. 4 (3), University of Chicago Press, 1986, pp. 376-414.

Cain, Glenn, "The challenge of segmented labor market theories to orthodox theory: a survey", Journal of Economic Literature, vol. 14, núm. 4, pp. 1215-1257.

Cimoli, Mario et al., "Cambio estructural, heterogeneidad productiva y tecnología en América Latina" en Mario Cimoli. (ed.) Heterogeneidad estructural, asimetrías tecnológicas y crecimiento en América Latina, Santiago de Chile, CEPAL-BID, 2005, pp. 9-37.

Vol. 40, núm. 158, julio-septiembre / 2009 
De Soto, Hernando, El otro sendero, $6^{\mathbf{a}}$ ed., Lima, Instituto Libertad y Democracia, 1987.

Dickens, William y Kevin Lang, "A test of dual labor market theory", en American Economic Review, vol. 75, núm. 4, Pittsburgh, 1985, pp. 792-805.

Diez de Medina, Rafael y Pascual Gerstenfeld, "Sector informal urbano: marco teórico, cuantificación y propuesta de medición para el caso uruguayo", Documento núm. 19, Primeras Jornadas Anuales de Economía, Montevideo, BCU, 1986.

Filgueira, Fernando y Denisse Gelber, "La informalidad en Uruguay: ¿un mecanismo de adaptación del trabajo o del capital?", Documento de Trabajo del IPES, Colección Monitor Social del Uruguay, núm. 5, Montevideo, Universidad Católica del Uruguay, 2003.

Lay, Jann, Informality and Segmentation in the Bolivian Labour Market: Empirical Evidence and Policy Implications, Instituto de Investigaciones Socioeconómicas, Universidad Católica Boliviana, Proyecto PIEB, enero de 2003.

Maloney, William, "Are labor markets in development countries dualistic?", Policy Research Working Paper, 1941.

Naciones Unidas, La protección social de cara al futuro: Acceso, financiamiento y solidaridad, marzo de 2006, Santiago de Chile, Naciones Unidas-CEPAL.

Notaro, Jorge, "La calidad del empleo en el Uruguay 1984-2003”, Documento de Trabajo 01/05, Montevideo, Instituto de Economía, Facultad de Ciencias Económicas y de Administración, 2005.
Oficina Internacional del Trabajo (OIT), Employment, Incomes and Inequality. A Strategy for Increasing Productive Employment in Kenya, Ginebra, OIT, 1972.

Piore, Michael, "Notes for a theory of labor market segmentation", en Richard Edwards et al. (eds.), Labor market segmentation, Lexington, Ma, D. C. Heath, 1975.

Piore, Michael y Charles Sabel, The Second Industrial Divide: Possibilities for Prosperity, Nueva York, Basic Books, 1984.

Portes, Alejandro, En torno a la informalidad: ensayos sobre teoría y medición de la economía no regulada, México, Miguel Ángel Porrúa, 1995.

Portes, Alejandro y William Haller, "La economía informal", Serie políticas sociales 100, Santiago de Chile, Naciones Unidas. División de Desarrollo Social, CEPAL, noviembre de 2004.

Portes, Alejandro, Silvia Blitzer y John Curtis, "The urban informal sector in Uruguay: its internal structure, characteristics and effects", en World Development, vol. 14, núm. 6, Quebec, Elsevier, 1986, pp. 10651076.

Saint Paul, Gilles, Dual Labor Markets: A Macroeconomic Perspective, Londres, MIT Press, 1996.

Tokman, Víctor, "Las relaciones entre los sectores formal e informal", en Revista de la CEPAL, núm. 5, primer semestre, Santiago de Chile, 1978, pp. 103-141.

, De la informalidad a la modernidad, Boletín Cinterfor, núm. 155, diciembre de 2003, Montevideo, Cinterfor, pp. 9-31. 


\section{Anexo}

\section{Cuadro A-1. Variables incluidas en los modelos probit}

\begin{tabular}{|c|c|}
\hline Variable & Especificación \\
\hline Sexo & Vale uno para las mujeres, cero para los hombres \\
\hline Edad & Expresada en tramos (14-17, 18-24, 25-44, $45-64$ y 65 y más), se omite el tercer tramo \\
\hline Relación de parentesco & Variables binarias que distinguen entre cónyuge, hijo y otro pariente, omitiendo a los jefes de hogar \\
\hline Localización geográfica & Vale uno si el trabajador reside en Montevideo, cero si reside en el interior urbano \\
\hline $\begin{array}{l}\text { Proporción de personas dependientes } \\
\text { en el hogar }\end{array}$ & Cociente entre los inactivos sin ingresos del hogar y el total de miembros del hogar \\
\hline Recibe jubilación & Variable binaria que indica si el trabajador percibe una jubilación \\
\hline Otros ingresos del hogar & Logaritmo del resto de los ingresos (per cápita) del hogar, se incluye también su expresión cuadrática \\
\hline $\begin{array}{l}\text { Existencia de otros aportantes en el } \\
\text { hogar }\end{array}$ & Vale uno si existen otros trabajadores que aportan a la seguridad social en el hogar, cero en otro caso \\
\hline Asistencia al sistema educativo & Vale uno si el trabajador asiste al sistema educativo, cero en otro caso \\
\hline Nivel educativo & $\begin{array}{l}\text { Variables binarias para los distintos niveles, se omite el primer tramo correspondiente a educación } \\
\text { primaria (completa e incompleta) }\end{array}$ \\
\hline Jornada a tiempo completo & $\begin{array}{l}\text { Variable binaria que indica si el trabajador tiene una jornada a tiempo completo } \\
\text { (más de } 35 \text { horas semanales) }\end{array}$ \\
\hline Mutiempleo & Variable binaria que indica si el trabajador posee más de un empleo \\
\hline Ramas de actividad & Variables binarias que reflejan la rama CIIU3 a un dígito, omitiendo la industria manufacturera \\
\hline Servicio doméstico & Variable binaria que distingue a los trabajadores del servicio doméstico \\
\hline Directivo & Variable binaria que distingue a los trabajadores que ocupan cargos directivos \\
\hline Microempresa & Variable binaria que indica si el trabajador trabaja en una empresa de menos de cinco empleados \\
\hline Funcionario público & Variable binaria que distingue a los asalariados públicos \\
\hline
\end{tabular}

\section{Desarrollo}

